\title{
Simulation of Quantum Cryptography and use of DNA based algorithm for Secure Communication
}

\author{
Sharvari Gogte ${ }^{1}$, Trupti Nemade ${ }^{2}$, Shweta Pawar ${ }^{3}$, Prajakta Nalawade ${ }^{4}$ \\ 1-4(B. Tech, Computer Technology Department, Veermata Jijabai Technological Institute (V.J.T.I.)/ Mumbai \\ University, India)
}

\begin{abstract}
Quantum Cryptography $(Q C)$ is an emerging security technique in which two parties communicate via a quantum channel. The fundamentals of quantum cryptography are No-cloning theorem and Heisenberg's uncertainty principle. This research paper proposes a simulation of quantum key exchange and authentication followed by an implementation of DNA based algorithm for secure message exchange.
\end{abstract}

Keywords: BB84 protocol, DNA based algorithm, Quantum Cryptography $(Q C)$, Secure key exchange by simulation

\section{Introduction}

Traditional cryptosystem has been in use for many years. It includes public as well as secret key cryptography. With the advent of supercomputers, even the computationally intensive algorithms like RSA, triple DES, AES, RC4, etc. are easily breakable. In secret key cryptography, secure key exchange through public channel is difficult. One time pad technique which was widely used and was proved to provide immense security fails to generate unique random keys after certain period of time. These shortcomings of classical cryptography led to the development of QC. ([1], [2])

QC is based on laws of physics namely No-cloning theorem and Heisenberg's uncertainty principle. ([2], [3])

\section{No-cloning theorem:}

It states that an exact copy of a quantum sized particle cannot be made. An attempt of reproducing the quantum particle would not result in exact copy of its true state. Due to this property of photons (a quantum sized particle), replay attack is prevented. An attempt to reproduce the current key will be detected, resulting in termination of communication.

\section{Heisenberg's Uncertainty principle:}

It states that at a given time, measuring the velocity and the location of a quantum sized particle is impossible. Hence, an eavesdropper cannot measure the stream of transmitted photons accurately. This principle also helps communicating parties to determine whether the stream of photons has been modified or not.

Based on these principles, the quantum channel is resistant to attacks like replay attack, man-in-themiddle attack, sniffing attack etc.

In order to implement $\mathrm{QC}$, we require fiber optic cables for transmission, photon generator and photon polarizer to generate polarized stream of photons. Since the hardware requirements are very expensive, the real life implementation of QC is limited. Hence we propose the simulation of QC. ([4])

We have used QC for authentication and secure key exchange followed by message encryption using DNA based algorithm. This algorithm is based on the central dogma of molecular biology which describes the flow of generic information within a biological system. Dogma provides a framework for understanding transfer of sequence information in living organisms containing sequence information carrying biopolymers such as DNA, RNA and Protein. As per the normal flow of biological information, DNA can be copied to DNA by DNA replication. Information can be copied into mRNA by transcription and proteins can be synthesized using the information in mRNA as a template by translation. ([5], [6])

\section{Transcription:}

It's the process that creates a new RNA piece by transferring information from a section of DNA strand. A DNA segment is read, non-coding areas are removed, and the remaining ones are re-joined and transcribed into a single strand of RNA.

\section{Translation:}

The RNA sequence is translated first, into a sequence of amino acids, then into a protein, according to the genetic code table. This central dogma framework is simulated in DNA module of our encryption algorithm. (Fig. 1) 


\section{Proposed System}

We have proposed a secure message transfer protocol which consists of the following modules.

\subsection{BB84 Protocol}

This protocol was invented by Charles Bennett and Gilles Brassard in 1984.The description of protocol is as follows: (Fig. 2) ([7] - [12]) (A and B are communicating parties)

1. A sends a stream of polarized photons to $\mathrm{B}$. The photons can be polarized in rectilinear $\left(0^{\circ}\right.$ or $\left.90^{\circ}\right)$ or diagonal direction $\left(45^{\circ}\right.$ or $\left.135^{\circ}\right)$. A stream of photons is string of zeros and ones with direction specified for each bit.

2. B measures this stream of photons with the help of randomly selected basis i.e. rectilinear or diagonal, and records the results.

Note: It is not necessary that A and B will use same basis for measurement.

3. B informs A, his basis used for measurement of photons, through public channel.

4. A then compares the received basis with the actual basis and informs B over the public channel, the correct bits.

Note: The correct bits are those whose basis are same.

5. Then A and B discard the incorrect bits and the correct ones are considered as a key.

\subsection{Authentication}

The authentication protocol makes use of classical as well as quantum cryptographic protocols. Since our main focus is on quantum cryptography, we have assumed that the identities of the communicating parties have already been established with a Third Party authentication server via classical cryptographic protocols through public channel. The procedure stated below describes how A and B authenticate to each other. (Fig. 3) ([13])

1. Initially, when A wishes to communicate with $\mathrm{B}, \mathrm{A}$ sends its own identity and the identity of $\mathrm{B}$ (the identity of the party with which the communication is intended) to authentication server via public channel.

2. After verifying the identities of A and B, the authentication server (AS) generates a random stream of basis and sends it to both the parties.

3. The AS then generates a random stream of photons, sends it to A and its complement to B.

4. A then sends a subset of received photons to B, and B checks whether he has complement of the bits or not.

5. After this successful verification, B repeats the same procedure but sends a disjoint stream of photons. Thus, A and B have authenticated themselves to each other.

\subsection{Secure Key Exchange}

We have implemented the secure key exchange module using the BB84 protocol ([7] - [12]) simulation. All the steps of the protocol are followed as-is. The only difference is in the method by which random bits and basis are generated.

A random stream of bits (zeroes and ones) which represents the stream of photons is generated as follows. To get $\mathrm{n}$ random bits, the function 'random' (which returns a random value between 0 and 1 ) is called $\mathrm{n}$ times. For a particular call, if the 'random' function returns a value less than or equal to 0.5 , then that particular bit is considered ' 0 ' else it is considered ' 1 '.

The method for generating the corresponding basis is similar. The only difference is in the last step. If the 'random' function returns a value less than or equal to 0.5 , then that particular basis is considered ' + ' else it is considered ' $\mathrm{x}$ '.

\subsection{DNA based algorithm}

The DNA based algorithm is symmetric block cipher. The inputs to this algorithm are a 128 bit key and plain text divided into blocks of 128 bits. ([6])

\subsubsection{Encryption}

The encryption procedure is as follows:

1. The user gives input in the form of a string which is then converted to its hexadecimal equivalent. If the size of this hexadecimal equivalent is less than 32 (128 bits /4) hexadecimal digits, then a padding of zeros is added.

2. Now these hexadecimal digits are transferred into $4 \mathrm{X} 4$ byte matrix $\mathrm{M}$.

3. The key exchanged in the 'secure key exchange' step is used to generate further 10 sub-keys $\left(\mathrm{K}_{0}-\mathrm{K}_{9}\right)$ with the help of AES sub-keys generator. 
4. XOR operation is performed between matrix $\mathrm{M}$ and first sub-key $\mathrm{K}_{0}$.

5. Then the plain text undergoes 8 rounds of transformation based on the DNA module.

6. The result obtained is then XORed with the last key $\left(\mathrm{K}_{9}\right)$ and thus we get the encrypted text.

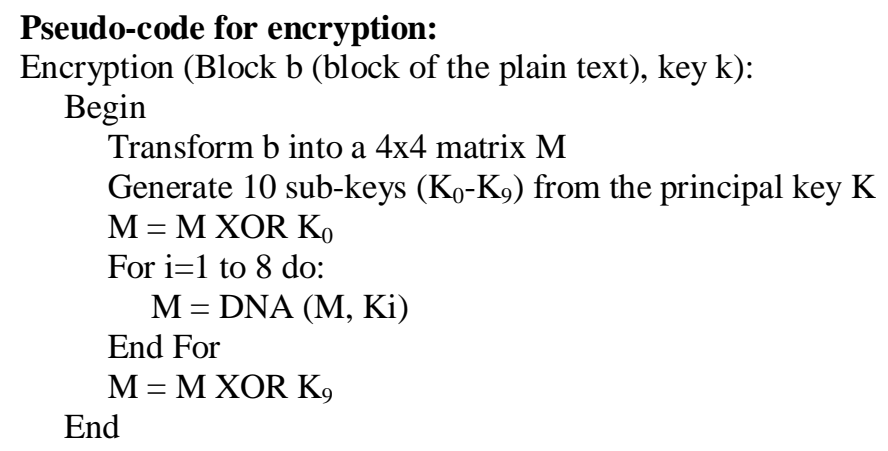

\subsubsection{DNA Module}

The DNA module treats every line of matrix $\mathrm{M}$ and the round keys as a DNA strand (A for $00, \mathrm{C}$ for $01, \mathrm{G}$ for 10 and $\mathrm{T}$ for 11$)$. Then the following three steps are applied:

\section{Transcription:}

This process simulates the transcription process of the central dogma. This is mono-alphabetic substitution. A DNA strand is converted to RNA strand by changing A to T, C to G, G to C and T to A.

\section{BIO_XOR:}

Given a Matrix $\mathrm{M}$ and a sub-key $\mathrm{K}_{\mathrm{i}}$, we compute M BIO_XOR $\mathrm{K}_{\mathrm{i}}$ using BIO_XOR (TABLE 1)

\section{Translation:}

This process simulates the translation of the central dogma which is also a substitution process performed using amino acid table (TABLE 2). The value in each block of matrix is divided into left and right part which is then used to look up the amino acid table for substitution.

\subsubsection{Decryption}

The steps for decryption are reverse of the encryption algorithm.

1. Initially, the encrypted matrix is XORed with the last key.

2. Then the matrix undergoes eight rounds of DNA module with the round keys.

3. The matrix so obtained is XORed with the first key to get the block of plaintext.

4. This block is read row wise to get back the original text.

The transcription and the BIO-XOR steps of the DNA module remain the same. For the translation process, an inverse of the amino acids translation table is made and then the lookup takes place.

For creating the inverse table, we traverse the original table row wise. The value obtained by reading the entry is divided into left and right half which transforms into the row and column index of the inverse matrix respectively. At that position, the concatenation of the row and column indices of the entry is inserted. This procedure is repeated for all the entries of the table. Thus we get the inverse amino acids table.

For example, if aminoAcid[AA,AT] = TAAT, then inverseAminoAcid[TA,AT] = AAAT

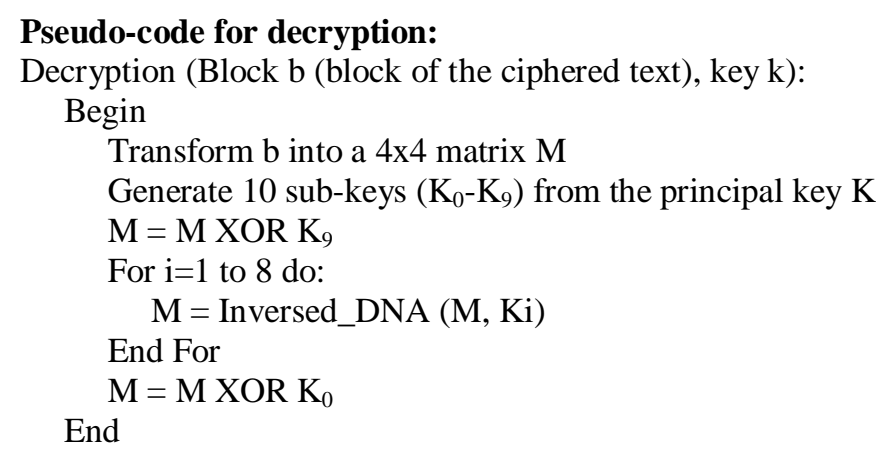

\section{Methods and Results}

The following are the screenshots of our implemented system along with the space and time complexity.

Time and space complexity: 
The space complexity of our system is $\mathrm{n}^{3}$ as we need to store ten sub-keys used for encryptiondecryption in a 3-dimensional array. (An array of 10 keys which are in a matrix form) The time complexity of our system is $\mathrm{n}^{3}$ since the encryption and decryption operations are applied on three dimensional arrays.

According to the System calculated time, the total time required for authentication, key-exchange and encryption-decryption is approximately 10 seconds. (Fig. 4-7) ([14])

\section{Figures and Tables}

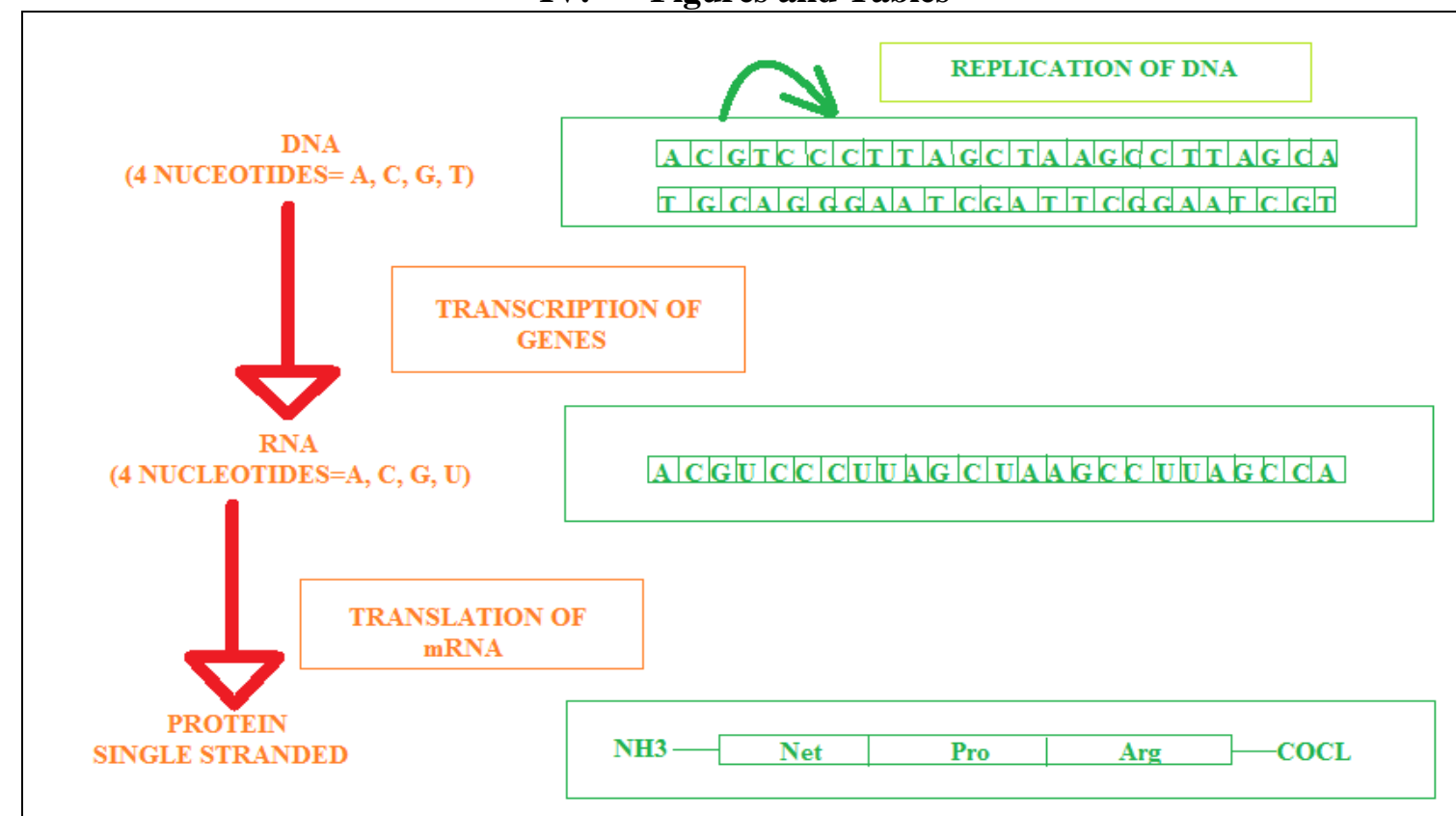

Fig. 1: central dogma of molecular biology ([5])

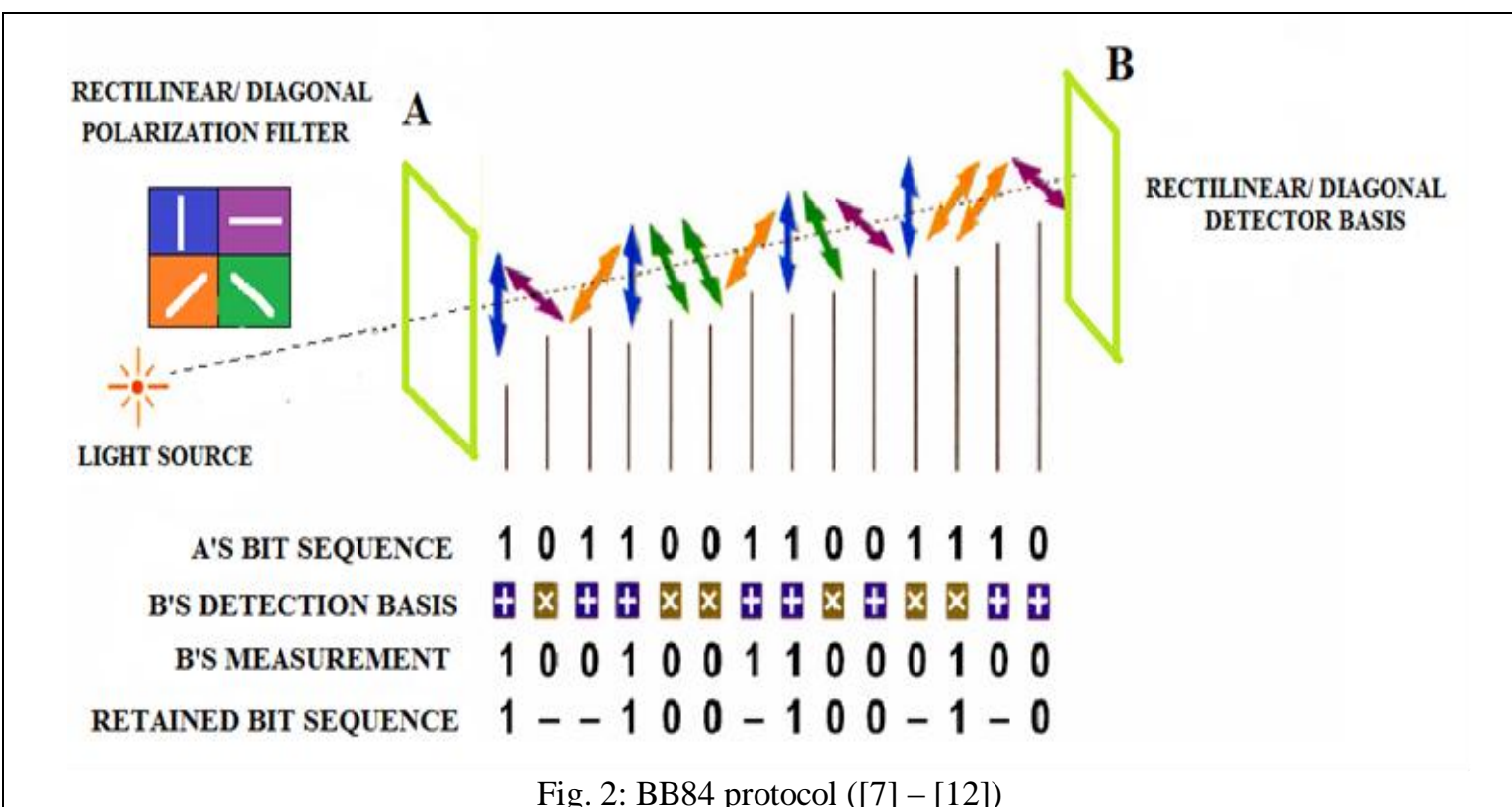

Fig. 2: BB84 protocol ([7] - [12]) 


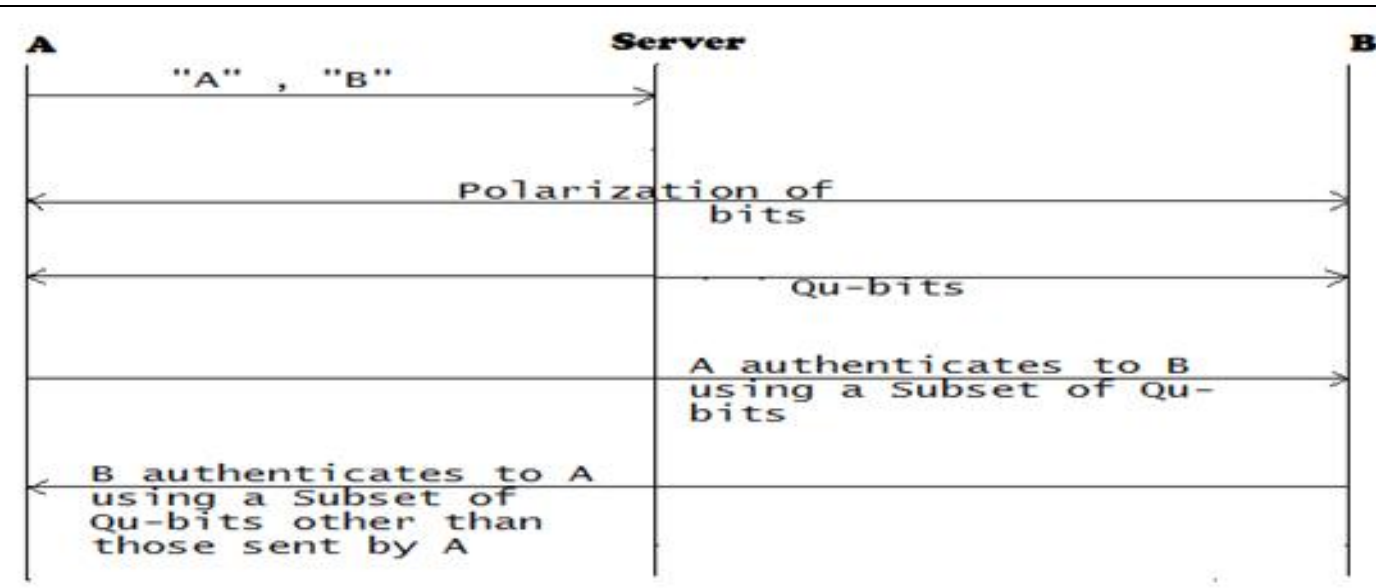

Fig. 3: authentication protocol ([13])

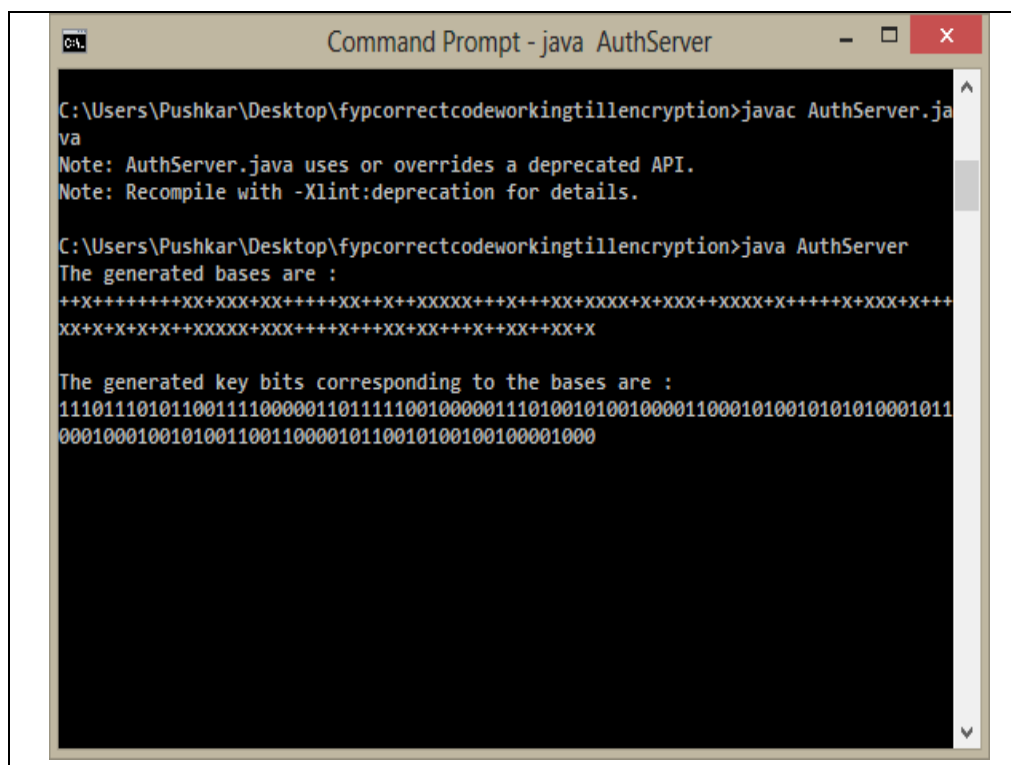

Fig. 4: authentication server 


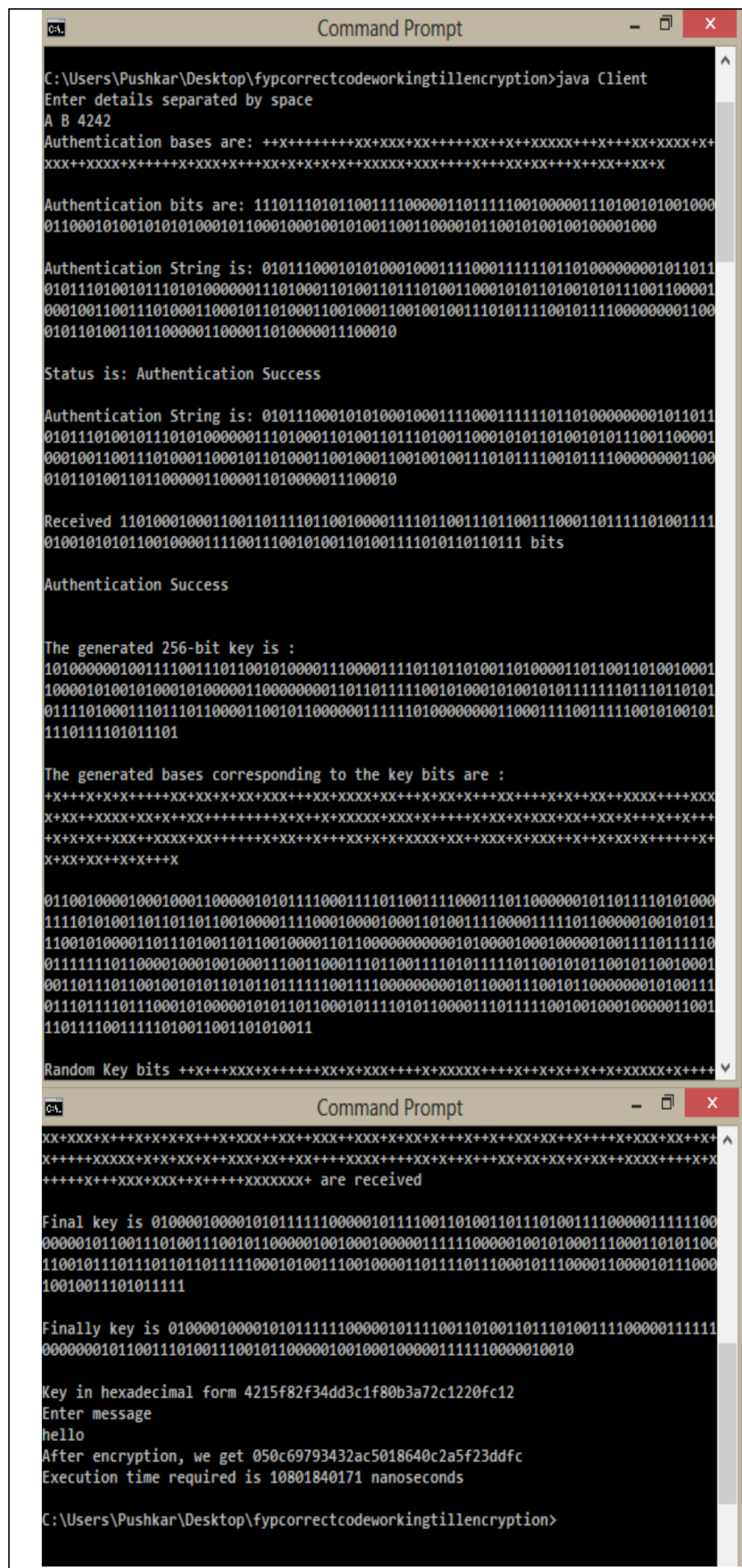

Fig. 5 and 6: communicating party A 


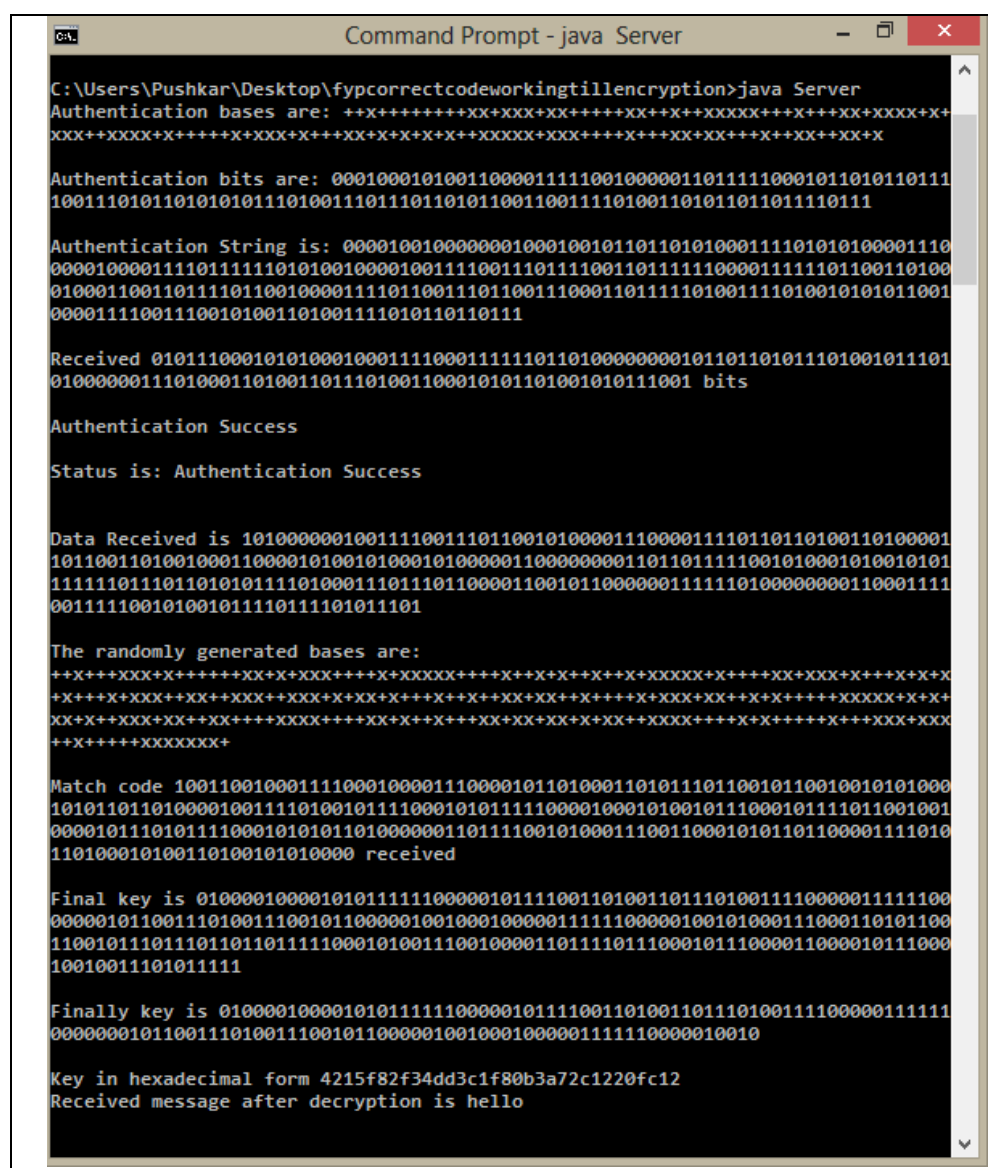

Fig. 7: communicating party B

\begin{tabular}{|l|l|l|l|l|}
\hline \multicolumn{5}{|c|}{ TABLE 1: BIO_XOR ([6]) } \\
\hline BIO_XOR & A & C & G & T \\
\hline A & T & G & C & A \\
\hline C & G & T & A & C \\
\hline G & C & A & T & G \\
\hline T & A & C & G & T \\
\hline
\end{tabular}

\begin{tabular}{|c|c|c|c|c|c|c|c|c|c|c|c|c|c|c|c|c|}
\hline \multicolumn{17}{|c|}{ TABLE 2: Amino Acids ([6]) } \\
\hline & AA & $A C$ & $A G$ & AT & CA & $C C$ & CG & CT & GA & $G C$ & GG & GT & TA & TC & TG & $\pi$ \\
\hline AA & TAAA & TAAC & TAAG & TAAT & TACA & TACC & TACG & TACT & TAGA & TAGC & TAGG & TAGT & TATA & TATC & TATG & TATT \\
\hline$A C$ & TCAA & TCAC & TCAG & TCAT & TCCA & TCCC & TCCG & TCCT & TCGA & TCGC & TCGG & TCGT & TCTA & TCTC & TCTG & TCT \\
\hline$A G$ & TGAA & TGAC & TGAG & TGAT & TGCA & TGCC & TGCG & TGCT & TGGA & TGGC & TGGG & TGGT & TGTA & TGTC & TGTG & TGT \\
\hline AT & TTAA & TाAC & TTAG & TTAT & TाCA & TाтC & TTCG & ПтСт & TIGA & TTGC & TTGG & TाGT & TाTA & तार & TाTG & $\pi \pi$ \\
\hline$C A$ & GAAA & GAAC & GAAG & GAAT & GACA & GACC & GACG & GACT & GAGA & GAGC & GAGG & GAGT & GATA & GATC & GATG & GAT \\
\hline CC & GCAA & GCAC & GAAG & GCAT & GCCA & GCCC & GCCG & GCCT & GCGA & GCGC & GCGG & GCGT & GCTT & GCTC & GCTG & GCT \\
\hline CG & GGAA & GGAC & GGAG & GGAT & GGCA & GGCC & GGCG & GGCT & GGGA & GGGC & GGGG & GGGT & GGTA & GGTC & GGTG & GGT \\
\hline CT & GTAA & GTAC & GTAG & GTAT & GTCA & GTCC & GTCG & GTCT & GTGA & GTGC & GTGG & GTGT & GTTA & GTTA & GTTG & GITा \\
\hline GA & CAAA & CAAC & CAAG & CAAT & CACA & CACC & CACG & CACT & CAGA & CAGC & CAGG & CAGT & CATA & CATC & CATG & CATT \\
\hline GC & CCAA & CCAC & CCAG & CCAT & CCCA & $\operatorname{cccc}$ & CCCG & CCCT & CCGA & $\operatorname{ccGC}$ & CCGG & CCGT & CCTA & СCTC & СCTG & $\mathrm{CCT}$ \\
\hline GG & CGAA & CGAC & CGAGA & CGAT & CGCA & CGCC & CGCG & CGCT & CGGA & CGGC & CGGG & CGGT & CGTA & CGTC & CGTG & CGT \\
\hline GT & СTAA & СТAC & CTAG & СТАT & CTCA & СтСС & CTCG & СТCT & CTGA & CTGC & CTGG & СTGT & СТTA & СтTс & СтTG & СTाT \\
\hline TA & AAAA & AAAC & AAAG & AAAT & AACA & AACC & AACG & AACT & AAGA & AAGC & AAGG & AAGT & AATT & AATA & AATC & AATG \\
\hline TC & ACAA & ACAC & ACAG & ACAT & ACCA & ACCC & ACCG & ACCT & ACGA & ACGG & ACGC & ACGT & ACTA & ACTC & ACTG & ACT \\
\hline TG & AGAA & AGAC & AGAG & AGAT & AGCA & AGCC & AGCG & AGCT & AGGA & AGGC & AGGG & AGGT & AGTA & AGTC & AGTG & AGT \\
\hline$\pi$ & ATAA & ATAC & ATAG & ATAT & ATCA & ATCC & ATCG & ATCT & ATGA & ATGC & ATGG & ATGT & ATTA & ATTC & ATTG & АाT \\
\hline
\end{tabular}




\section{Conclusion}

Our system can guard against man in the middle attack, eavesdropping, spoofing, packet sniffing and replay attack. This is because the fundamentals of QC are based on the laws of physics; thus the eavesdropper would be unable to generate the exact replica of the photons exchanged between the communicating parties as well as record the stream of photons exchanged between the parties. These factors increase the security of our system. ([1], [2])

Our further research includes:

1. Overcoming distance limitations

2. Protection against denial of service attack

3. To devise cost effective methods for generation, polarization, transmission of photons and retaining their polarizations over long distances

\section{Acknowledgements}

We would like to thank our project mentor Prof. Seema Shrawne for guiding us throughout our project. Her suggestions have helped us in effective planning of our work and have added value to it. We appreciate the time that she invested in our project in spite of her busy schedule. We are grateful for her constant encouragement and co-operation which was instrumental in driving our project work.

\section{References}

[1] Karen Hunter, QuantumCryptography, SCI 510, Quantum Todd Duncan

[2] Sheila Cobourne , Quantum Key Distribution Protocols and Applications, Royal Holloway, University of London, Egham, Surrey TW20 0EX, England

[3] Kelly Koenig, Bleeding edge: Nano-technological application in Quantum Cryptography, CSC grants, December 2008

[4] Serge Massar, Fiber Optics Protocols for Quantum Communication, Quantum Information Processing, Vol. 5, No. 6, December 2006

[5] Wikipedia, http://en.wikipedia.org/wiki/Central_dogma_of_molecular_biology

[6] Souhila Sadeg, Mohamed Gougache, Nabil Mansouri, Habiba Drias, An encryption algorithm inspired from DNA,IEEE, 978-14244-8611-3/10, 2010

[7] Charles H. Bennett, Gilles Brassard, Quantum cryptography: public key distribution and coin tossing, International Conference on Computers, Systems and Signal Processing, Banglore, India, December 10-12, 1984

[8] Mehrdad S. Sharbaf, Quantum Cryptography: A New Generation of Information Technology Security System, 2009 Sixth International Conference on Information Technology: New Generations

[9] Mehrdad S. Sharbaf, Quantum Cryptography: An Emerging Technology in Network Security, IEEE, 978-1-4577-1376-7/11, 2011

[10] Marcin Sobota, Adrian Kapczy_ski, Arkadiusz Banasik, Application of Quantum Cryptography protocols in Authentication process, The 6th IEEE International Conference on Intelligent Data Acquisition and Advanced Computing Systems: Technology and Applications, 15-17 September 2011, Prague, Czech Republic

[11] Yoshito Kanamori, Seong-Moo Yoo, Don A. Gregory, Frederick T. Sheldon, On Quantum Authentication Protocols, IEEE GLOBECOM 2005 proceedings

[12] http://swissquantum.idquantique.com/?Key-Sifting

[13] D. Richard Kuhn, A quantum cryptographic protocol with detection of compromised server, Quantum Information and Computation, Vol. 5, No.7 (2005) 551-560

[14] Stack Overflow, http://stackoverflow.com/questions/6789385/calculation-of-execution-time 